\title{
A New lon Source and Procedures for Atmospheric Pressure-Electron Capture Dissociation of Peptides
}

\author{
Damon B. Robb, ${ }^{1}$ Jason C. Rogalski, ${ }^{2}$ Juergen Kast, ${ }^{1,2}$ Michael W. Blades ${ }^{1}$ \\ ${ }^{1}$ Department of Chemistry, University of British Columbia, Vancouver, B.C., V6T 1Z1, Canada \\ ${ }^{2}$ The Biomedical Research Centre, University of British Columbia, Vancouver, B.C., Canada
}

\begin{abstract}
We introduce a new atmospheric pressure-electron capture dissociation (AP-ECD) source in which conventional nanospray emitters are coupled with the source block and photoionization lamp of a PhotoSpray APPI source. We also introduce procedures for data collection and processing, aimed at maximizing the signal-to-background ratio of ECD products. Representative data from Substance $\mathrm{P}$ are presented to demonstrate the performance of the technique. Further, we demonstrate the effects of two important experimental variables, source temperature and vacuum-interface declustering potential (DP), on the method. Last, we show that even when a high source temperature is used to maximize efficiency, AP-ECD fragments of a model phosphorylated peptide retain the modification.
\end{abstract}

Key words: AP-ECD, Electron capture dissociation, Ion source, Peptides

\section{Introduction}

$\mathrm{E}^{\mathrm{k}}$ lectron capture dissociation (ECD) [1-4] and electron transfer dissociation (ETD) [5-7] are ion fragmentation techniques for the mass spectrometric analysis of peptides and proteins providing several key advantages over collision induced dissociation (CID). The advantages of ECD and ETD stem from how their radical-driven mechanisms lead to preferential cleavage of disulfide and $\mathrm{N}-\mathrm{C}_{\alpha}$ bonds in polypeptides [8], without randomization of energy in the molecule prior to fragmentation. Of these advantages, perhaps the greatest is that labile post-translational modifications are generally retained during fragmentation, permitting their direct localization, which is often impossible with CID [9]. Further, bond cleavage in both methods tends to be more evenly distributed along the peptide backbone than in

Electronic supplementary material The online version of this article (doi:10.1007/s13361-011-0202-0) contains supplementary material, which is available to authorized users.

Correspondence to: Damon B. Robb; e-mail: robb@chem.ubc.ca
CID, so that they yield more complete sequence coverage. Because of these and other performance characteristics, there is a large and growing body of researchers interested in making use of ECD/ETD; however, the wide-spread adoption of these techniques has been slowed by their prohibitive instrumentation requirements: $\mathrm{ECD}$ is generally limited to FT-ICR instruments, while ETD has been mostly performed on special ion trap instruments, which have only recently become commercially available and are expensive. In light of these circumstances, an alternate ECD/ETD method that does not require the acquisition of an entire new instrument, or substantial modification of an existing instrument $[10,11]$, would plainly be welcomed.

In-source atmospheric pressure-electron capture dissociation (AP-ECD) [12-19] is an emerging technique with the potential to be a less expensive, more accessible alternative to conventional in vacuo ECD/ETD methods, because it can be implemented using a stand-alone ion-source device, in principle suitable for use with any existing or future atmospheric pressure ionization mass spectrometer, without requiring modification of the main instrument. The first reports of an atmospheric pressure ECD/ETD method were 
from Laprévote's group [12, 13], who discovered that peptide ions could be fragmented by an ECD-like process within a PhotoSpray atmospheric pressure photoionization (APPI) source [20,21]; in these studies, peptide ions from the source's heated nebulizer were shown to undergo ECD/ETD reactions within the APPI source block, following the generation of photoelectrons from an ionizable dopant in the sample vapor stream. We have endeavored to build upon this early work, with the goal of developing AP-ECD into a practical tool for peptide and protein analysis. To this end, we first modified the heated nebulizer of a PhotoSpray source so that its internal pneumatic sprayer could be electrified, resulting in a roughly $100 \times$ increase in peptide ion yield [22]; with this modification, the sensitivity of AP-ECD using a PhotoSpray source was much improved [14], so that readily interpretable spectra could be obtained from 100 fmol sample consumed $[16,18]$. In parallel, Berkout and Doroshenko have also recently presented results for ECD-like peptide fragmentation at atmospheric pressure [17]; key differences of this work from the other examples are that the peptide ions to be fragmented were first mass selected by high-field asymmetric waveform ion mobility spectrometry (FAIMS) [23], and a corona discharge was used as the source of reagent electrons.

Here, we introduce a new AP-ECD source in which conventional nanospray emitters are coupled with the source block and photoionization lamp of a PhotoSpray source. We also introduce procedures for data collection and processing, aimed at maximizing the signal-to-background ratio of ECD products. Representative data from Substance P are presented to demonstrate the performance of the technique. Further, we demonstrate the effects of two important experimental variables, source temperature and vacuuminterface declustering potential (DP), on the method. Last, we show that even when a high source temperature is used to maximize efficiency, AP-ECD fragments of a model phosphorylated peptide retain the modification.

\section{Experimental}

\section{AP-ECD Source}

Figure 1 is a schematic of the AP-ECD source. Briefly, the source consists of three interconnected sub-assemblies: a sprayer plug with an integrated nanospray source, a spray chamber with a central channel and an inlet for an auxiliary (AUX) gas flow, and the source block with photoionization lamp of an AB SCIEX (Concord, ON, Canada) PhotoSpray APPI source [20, 21]; a detailed description of the source is provided in the Supplementary Material section. In operation, the nanospray source generates gas-phase peptide ions within the spray chamber from a liquid sample stream bearing one or more peptides. These ions are transported rapidly from the nanospray source by a flow of gas to a region within the source block which may be irradiated by the photoionization lamp. When the lamp is switched off, the peptide ions pass through unaffected, and the source functions as a normal nanospray source. When the lamp is switched on, photoelectrons are generated from a photoionizable species doped into the gas flow; the photoelectrons are subsequently captured by the peptide ions in the downstream reaction/transport zone, resulting in ECD, and the fragment ions pass through the vacuum interface and into the mass analyzer for detection.

\section{Chemicals and Sample Preparation}

Peptide RPKPQQFFGLM-NH $\mathrm{H}_{2}$ (Substance P) was from Sigma-Aldrich (Oakville, ON, Canada) and peptide KRPpSQRHGSKY-NH ${ }_{2}$ (UOM9, PKC Substrate, phosphorylated) was from AnaSpec (San Jose, CA, USA). Methanol (HPLC-grade) and formic acid (88\% in water) were from Fisher Scientific (Ottawa, ON, Canada). Deionized water was from an in-house generator. The dopant used to promote photoelectron generation was HPLC-grade acetone from Fisher Scientific. Liquid nitrogen boil-off was used for all source gases.

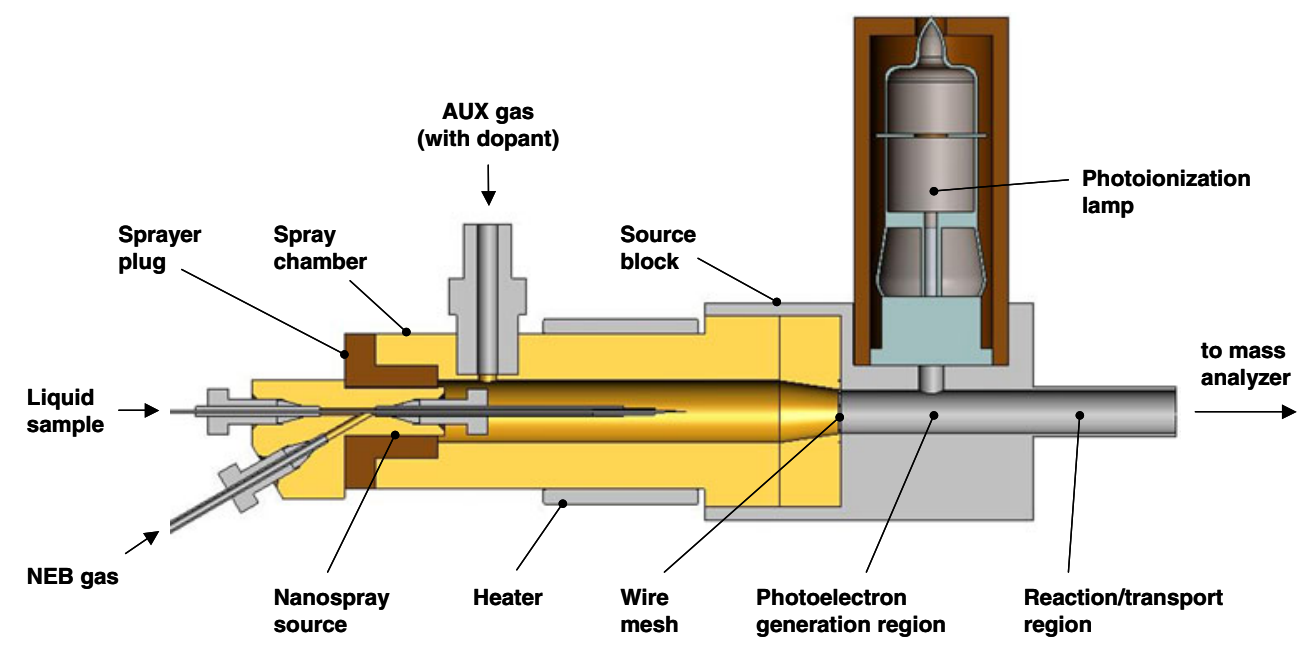

Figure 1. Schematic of the AP-ECD source 
For Substance P and UOM9, respectively, $200 \mu \mathrm{M}$ and $100 \mu \mathrm{M}$ stock solutions were prepared in water in polypropylene (PP) vials. For each peptide, a $4 \mu \mathrm{M}$ sample solution was prepared by diluting its stock solution in 50/50/0.05 methanol/ water/formic acid (vol/vol/vol) in a glass autosampler vial (PP vials were not used for the sample solutions to prevent their contamination with methanol-soluble plasticizers, which can be ionized in the source and thus raise the chemical background in the spectra).

\section{Instrumentation and Methods}

The mass spectrometer was a QSTAR XL Q-TOF instrument from AB SCIEX [24]. The sample solutions were infused at $0.4 \mu \mathrm{L} \mathrm{min}{ }^{-1}$ via the instrument's built-in syringe pump. The acetone dopant was delivered at $0.1 \mu \mathrm{L} \mathrm{min}^{-1}$ via a separate syringe pump. The nanospray source voltage, normally $2.2-2.5 \mathrm{kV}$, was provided by an external high voltage power supply (model PS 350; Stanford Research Systems; Sunnyvale, CA, USA). The spray chamber and APPI source block were held at $1.2 \mathrm{kV}$ via the Ion Source HV power supply of the mass spectrometer. The photoionization lamp was powered by the PhotoSpray control unit, which was switched on and off manually. The AUX and NEB gases were from the instrument's GS1 and GS2 outputs, respectively, set to 20 and 30 . The volumetric flow rate of the AUX gas was $9.2 \mathrm{Lmin}^{-1}$, measured using an inline mass flow controller (model $810 \mathrm{C}$; Sierra Instruments; Monterey, CA, USA). The APPI lamp gas was set to $0.6 \mathrm{Lmin}^{-1}$ using the PhotoSpray Control Unit's builtin flowmeter. The temperature (T) of the source's heater and the orifice-skimmer declustering potential (DP) and ring focusing potential (FP) of the instrument's vacuum interface $[24,25]$ were varied during the experiments; the $T$ and DP settings for each experiment are indicated in the text and the Figure captions; FP was always four times DP.

For data acquisition, the accumulation time per scan was $0.75 \mathrm{~s}$ and three time bins were summed per data point. The nanospray-only spectra (Figures $2 \mathrm{a}, \mathrm{b}$, and $3 \mathrm{a}$ ), and the raw AP-ECD spectra (Figures $2 \mathrm{c}$ and $3 \mathrm{~b}$ ), are each the sum of 5 scans, and so the sample consumption per spectrum was 100 fmol. The processed AP-ECD spectra (Figures 2d, e, 3c, 4,5 , and 6) are each the sum of 5 scans acquired with the lamp on, minus the sum of 5 scans with the lamp off, for 10 scans total, corresponding to $200 \mathrm{fmol}$ of sample consumed per spectrum. All the spectra have been centroided (peak finding threshold $=1$ count), except for Figures $2 \mathrm{a}-\mathrm{d}$ and 3 .

\section{Results and Discussion}

\section{Data Collection/Processing Procedures and Performance Demonstration}

Figure 2 presents a series of Substance $\mathrm{P}$ spectra illustrating the various steps of the data collection and processing procedures employed here; the final spectrum demonstrates the performance of the overall AP-ECD method.

Figure $2 \mathrm{a}$ is a survey scan showing the precursor ions to be subjected to AP-ECD, in this case mostly triply- and doubly-protonated molecular ions of Substance P. This spectrum was obtained with the photoionization lamp off and the interface declustering potential set to a relatively low level $(30 \mathrm{~V})$, conditions under which there is no ECD or substantial up-front CID in the vacuum interface, and so it closely reflects the ion population initially produced by the nanospray source. Though not strictly required for the method, this spectrum has been included because we expect that it may be advantageous to collect such precursor spectra during AP-ECD experiments, since they provide an unambiguous record of the species contributing to the final ECD spectra.

Figure $2 b$ shows a "lamp off" background spectrum acquired under the same conditions as Figure 2a, except that DP was increased to $100 \mathrm{~V}$, the value to be used for collecting the raw AP-ECD spectra. As demonstrated below, the use of high vacuum interface voltages can simplify the final spectra and improve the sensitivity for some ECD fragments; however, an additional consequence is that upfront ("nozzle-skimmer") CID may become significant [26, 27], particularly for the multiply-charged precursors. The effects of up-front CID are plainly evident in Figure 2b: compared with Figure $2 \mathrm{a}$, the $(\mathrm{M}+3 \mathrm{H})^{3+}$ ions are completely removed, the $(\mathrm{M}+2 \mathrm{H})^{2+}$ ions are substantially depleted, and there is an increased relative abundance of CID products, including numerous $a$ - and $b$-type fragment ions of Substance P. Note that the abundance of sodiated doublycharged molecular ions actually increases at the higher DP level, presumably because they are less susceptible to CID than protonated ions [26] and - absent CID - higher nozzleskimmer voltages improve ion transmission through the vacuum interface $[25,27]$. The CID products have the potential to obscure the ECD fragments in the AP-ECD spectra and to complicate data analysis. Fortunately, it is trivial to isolate the ECD products from the CID products by background subtraction, as illustrated below, provided lampoff background spectra such as Figure $2 \mathrm{~b}$ are collected along with the raw AP-ECD data.

Figure $2 \mathrm{c}$ is a raw AP-ECD spectrum acquired under the same conditions as Figure $2 b$, except that the photoionization lamp was switched on to generate electrons within the source for reaction with the precursor ions. Inspection of the spectrum reveals Substance P ECD fragments ( $c$ - and $z$-type ions) among a complex background including residual precursors as well as the abundant products of up-front CID noted above. Though there may be situations where it is desirable to obtain both ECD and CID products in the same spectrum, as occurs under the experimental conditions used here, it is certainly desirable to have the option to view the ECD products in isolation, to facilitate data interpretation. Two post-acquisition data processing steps, background subtraction and centroiding, are available to help achieve this. 
(a)

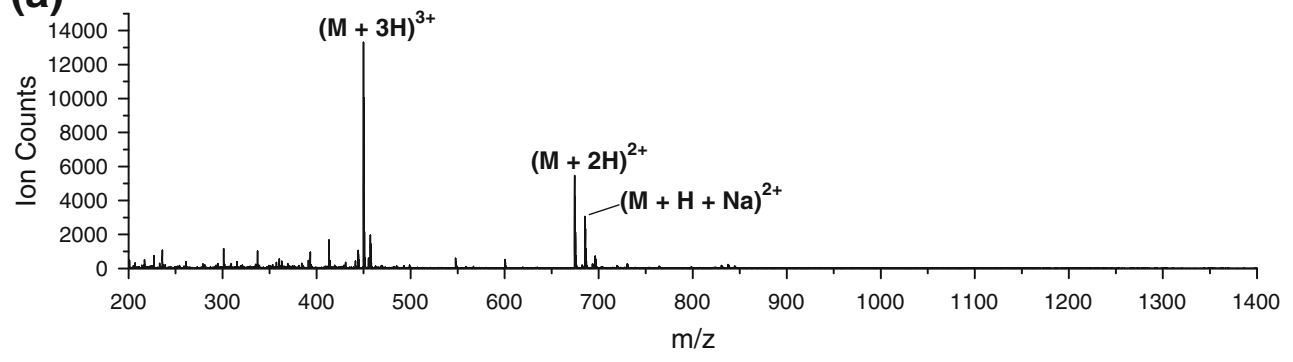

(b)
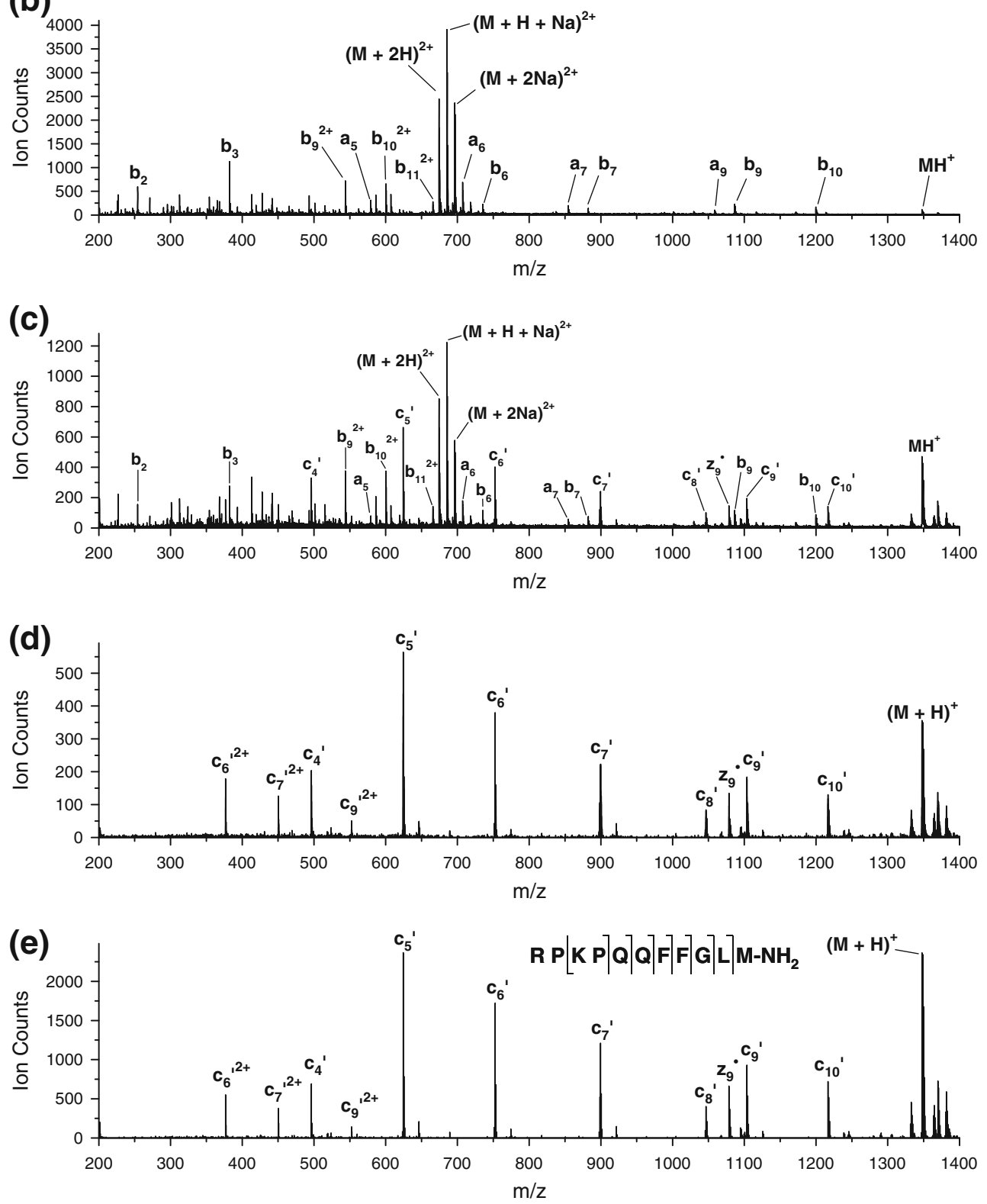

Figure 2. Spectra of Substance $P$ illustrating the various steps of the AP-ECD data collection and processing procedures, as well as demonstrating the performance of the overall method: (a) Precursor spectrum (lamp off, DP=30 V); (b) Background spectrum (lamp off, DP=100 V); (c) Raw AP-ECD spectrum (lamp on, DP=100 V); (d) Background subtracted AP-ECD spectrum, (c)-(b); (e) final AP-ECD spectrum, (d) centroided. ( $T=100{ }^{\circ} \mathrm{C}$ for all) 
(a)

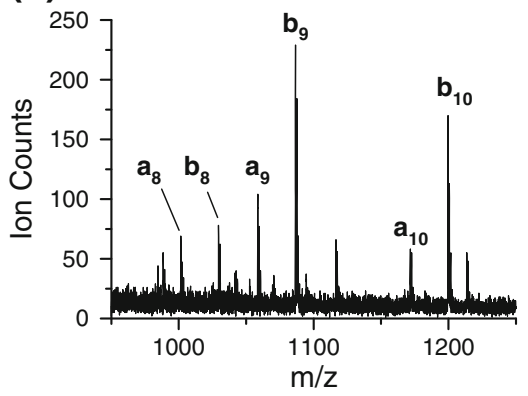

(b)

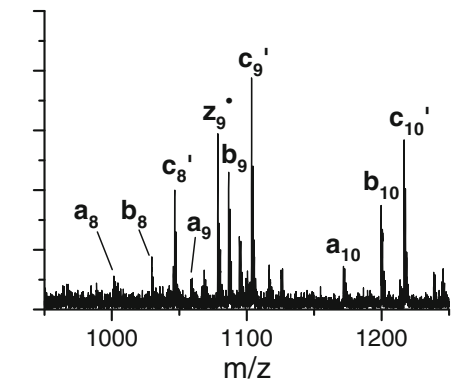

(c)

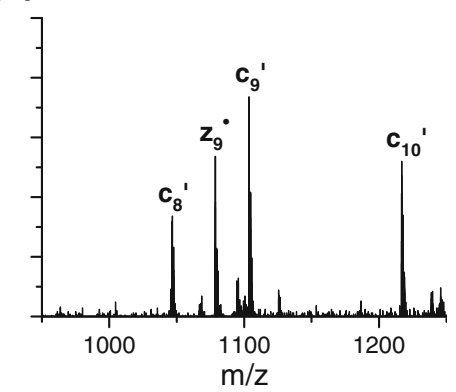

Figure 3. Expanded versions of Figure $2 b-d$ showing details of the background subtraction procedure (see Figure 2 caption and the text)

Figure $2 \mathrm{~d}$ is the spectrum obtained from the raw AP-ECD spectrum (Figure 2c) after subtraction of the lamp-off background spectrum (Figure 2b). The background subtraction procedure is effective, as the resulting spectrum consists nearly entirely of ECD products, with the residual precursors and CID products of the raw spectrum being removed down to a low level of baseline noise.

Figure $2 \mathrm{e}$ is the final AP-ECD spectrum, obtained by centroiding Figure 2d. Centroiding is advantageous here because-while binning the counts from a given ion peak into a single channel, increasing peak height and compressing the data - its algorithm discriminates against improbable asymmetric peak shapes, and thereby removes many spurious noise signals and improves the signal-to-background ratio in the final spectrum.

The final AP-ECD spectrum demonstrates the performance of the overall method, and it shows a prominent series of $c$-type fragment ions, $c_{4}{ }^{\prime}-c_{10}{ }^{\prime}$, as well as a single $z$-type fragment, $z_{9}{ }^{\circ}$, at high intensities relative to the background [28]. ${ }^{1}$ Of the expected ECD fragments for Substance P [29], only $c_{2}$ ' is missing, presumably because under the conditions of the experiment it is collisionally dissociated in the vacuum interface (see the section on the effects of DP, below). Also evident in the spectrum are minor peaks due to sodiated $c$-type fragments (unlabeled), as well as doublycharged ECD fragments, $c_{6}{ }^{2+}, c_{7}{ }^{2+}$, and $c_{9}{ }^{2+}$. Absent from the spectrum are the residual multiply-charged precursors typically observed in ECD/ETD spectra; this is an artifact of the background subtraction procedure, discussed further below. Altogether, with strong signals from ECD fragments and a low background, Figure 2e indicates that the performance of AP-ECD with the new source and data collection/

\footnotetext{
${ }^{1}$ Notation for ECD fragments follows the scheme introduced by Kjeldsen et al. [28], with homolytic N-C $\alpha$ bond cleavage assumed and with extra hydrogens on fragments explicitly indicated by apostrophes, "; note that in our previous paper [18], $z_{9}{ }^{\circ}$ at $\mathrm{m} / \mathrm{z} 1078.6$ was mistakenly assigned as $\mathrm{z}_{9}{ }^{\prime}$, with an extra hydrogen, as the result of our unwitting use of a peptide fragment calculator that provided $\mathrm{m} / \mathrm{z}$ values for $z$ ions instead of $z^{*}$ ions, which are $1 \mathrm{Da}$ heavier (the same mistake was made for all $z^{*}$ ions in the previous paper).
}

processing procedures is such that it can yield quality data from low fmol sample quantities.

To illuminate details of the method impacting the background subtraction procedure, Figure $3 \mathrm{a}-\mathrm{c}$ present expanded versions of Figure $2 \mathrm{~b}-\mathrm{d}$, respectively, focusing on the region of $m / z$ 950-1250. Figure 3a is the lamp-off background spectrum showing two partial series of $a$ - and $b$-type CID fragments of Substance P, and also a continuum of nanospray-generated ions that was not readily apparent in the full scale spectrum; this background continuum is significant because it can obscure weak ECD fragment peaks and raise
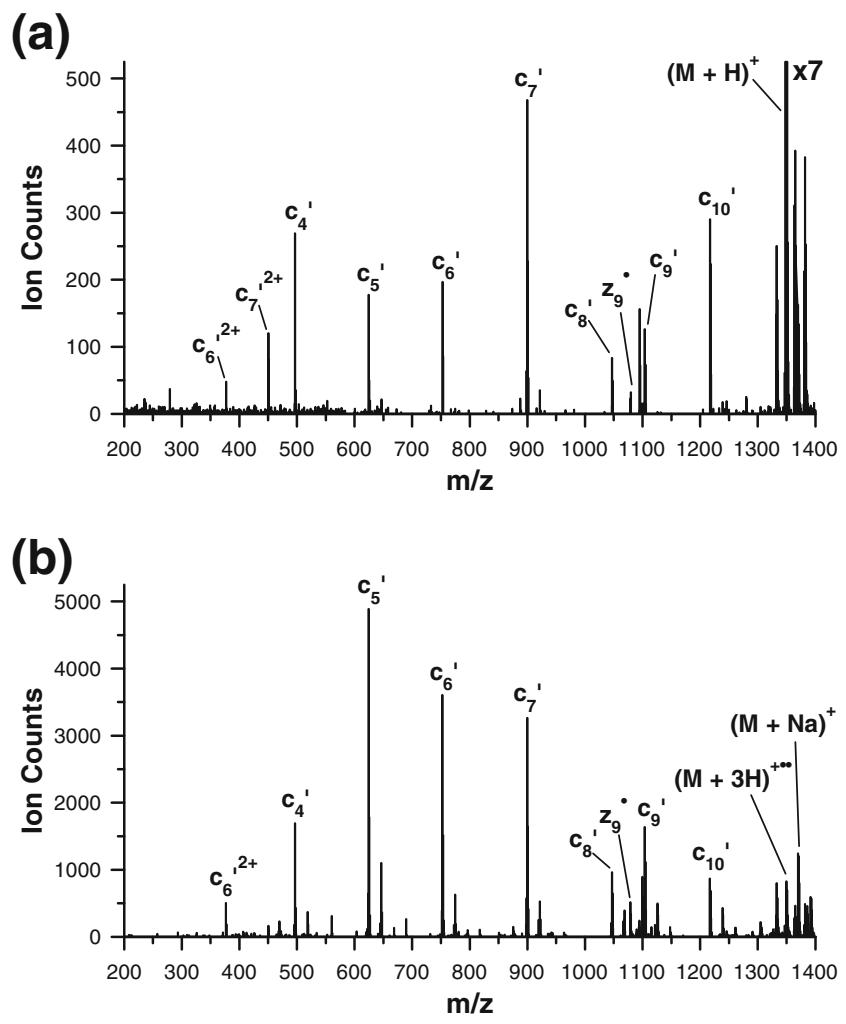

Figure 4. AP-ECD spectra of Substance $P$ showing the effects of the source temperature: (a) $\mathrm{T}=$ room temperature $\left(\sim 20^{\circ} \mathrm{C}\right)$; (b) $\mathrm{T}=200^{\circ} \mathrm{C}$. (DP=100 V for both) 
(a)

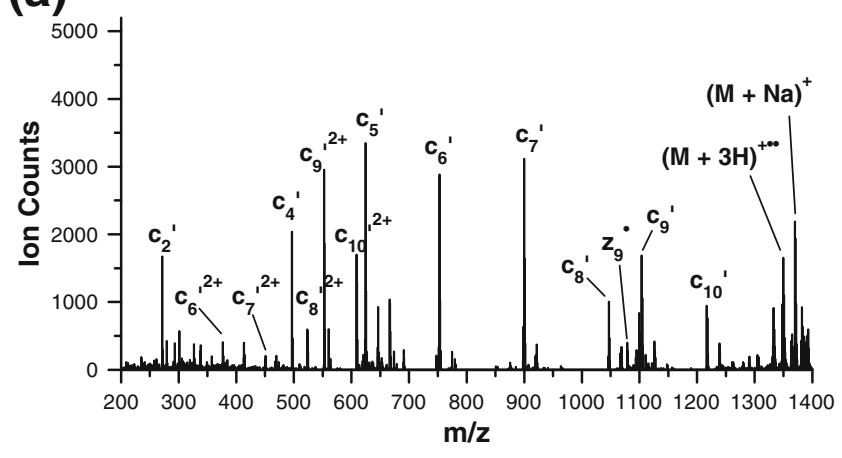

(b)

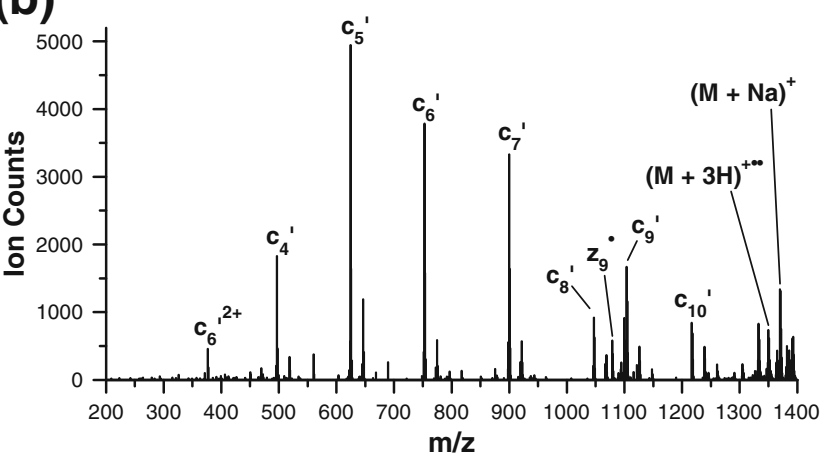

Figure 5. AP-ECD spectra of Substance $P$ showing the effects of the vacuum interface declustering potential: (a) $D P=$ $60 \mathrm{~V}$; (b) $\mathrm{DP}=100 \mathrm{~V}$. (T=200 ${ }^{\circ} \mathrm{C}$ for both)

the detection limits of the method. Figure $3 \mathrm{~b}$ is the raw APECD spectrum showing the effects of electron production: $c$ - and $z$-type ECD fragments of Substance $\mathrm{P}$ are generated and, in addition, both the CID products and the nanospray ionization continuum are reduced in intensity. The latter phenomenon is due to neutralization of background ions by electron capture and - importantly - it ensures that when the lamp-off background spectrum is subtracted from the raw AP-ECD spectrum, the baseline of the resulting spectrum is mostly negative except for where ECD products are present; this permits very clean spectra of the ECD products to be acquired, even from raw AP-ECD spectra having a complex background. Last, Figure 3c, the background-subtracted AP-ECD spectrum confirms that the background continuum and CID products are well-removed by the procedure, with only ECD products and a residue of baseline noise remaining. (Note that the precursor ion signals are also reduced in intensity by the electron capture process, of course, and so the background subtraction procedure also removes the residual precursor peaks, accounting for their absence from Figure $2 \mathrm{~d}$ and e.)

\section{Effects of Temperature}

Figure $4 \mathrm{a}$ and $\mathrm{b}$ show AP-ECD spectra of Substance P acquired with the source heater at room temperature and at $200{ }^{\circ} \mathrm{C}$, respectively. The yield of ECD fragments is increased substantially at the higher temperature, by an average factor of about twelve (though quality results are obtainable at room temperature, albeit at lower sensitivity and signal-to-noise). The increased yield of ECD fragments at $200{ }^{\circ} \mathrm{C}$ is attributable in part to an increase in peptide ionization efficiency, as the intensities of the multiplyprotonated Substance P precursor ions are roughly doubled at the higher temperature (results not shown), and it is reasonable to assume that an increased abundance of precursor ions will provide a proportional increase in ECD fragments. Also, the results suggest that there is a competitive proton transfer reaction pathway active primarily at lower temperatures, as evidenced by the very large $(\mathrm{M}+\mathrm{H})^{+}$ peak in Figure $4 a$, absent from Figure $4 b$; though it is premature to speculate on the mechanism responsible for this pathway, it is plainly inhibited by raising the source temperature, as ECD is by far the dominant process at $200{ }^{\circ} \mathrm{C}$. As for the effects of temperature on ECD itself, we presume that heating the source serves the same function as the various heating methods employed in "activated ion" ECD [30-32] and ETD [33, 34], where the internal energy of precursor ions is elevated prior to electron capture to increase conformational heterogeneity and, thereby, to increase sequence coverage, improve yield, and reduce the extent of hydrogen-atom migration [32, 34]. The results of Figure 4 appear to support this hypothesis generally. Moreover, as noted by a reviewer, there is a change in the relative abundance of the various fragments with temperature, with $c_{7}$ and $c_{10}{ }^{\prime}$ predominating at relatively low temperature only, which is consistent with prior temperature-dependent ECD results for Substance P, where $c_{7}{ }^{\prime}$ and $c_{10}{ }^{\prime}$ were the sole ECD fragments observed at $86 \mathrm{~K}$ [31].

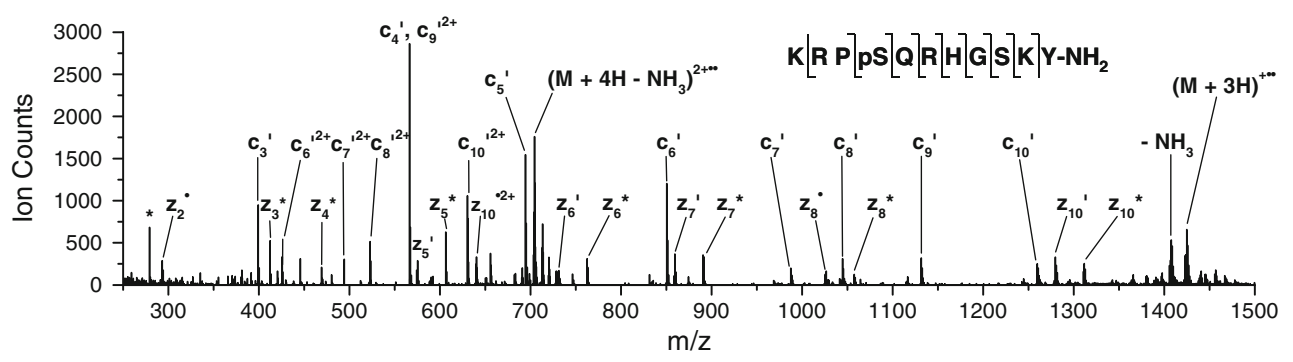

Figure 6. AP-ECD spectrum of the phosphopeptide UOM9. $\left(T=200{ }^{\circ} \mathrm{C}, \mathrm{DP}=40 / 80 \mathrm{~V}\right)$ 


\section{Effects of Declustering Potential}

Figure $5 \mathrm{a}$ and $\mathrm{b}$ show AP-ECD spectra of Substance $\mathrm{P}$ acquired at $\mathrm{DP}=60$ and $100 \mathrm{~V}$, respectively. At $\mathrm{DP}=60 \mathrm{~V}$, the lower $\mathrm{m} / \mathrm{z}$ portion of the spectrum is complex, with the $c_{2}{ }^{\prime}$ fragment of Substance $\mathrm{P}$ being identifiable among numerous doubly-charged fragments, including $c_{6}{ }^{12+}-c_{10^{12+}}$, and also a sizable chemical background due to solvent and dopant impurities (which are ionized through ion-molecule reactions stemming from the photoionization of acetone and are not removed by the background subtraction procedure used here). At $\mathrm{DP}=100 \mathrm{~V}$, the spectrum is simpler and easier to interpret, as the doubly-charged fragments and chemical background species are mostly eliminated by up-front CID in the vacuum interface $[26,27]$; however, the $c_{2}{ }^{\prime}$ fragment is also removed, indicating that there can be a trade-off between spectral simplicity and sequence coverage when the voltages in the vacuum interface are raised. Though not readily apparent from the limited data of Figure 5, note that DP also affects sensitivity substantially, with the intensity of each ECD fragment generally increasing with DP over a broad range, until the onset of up-front CID, with its optimum DP value being a function of both its $\mathrm{m} / \mathrm{z}$ and its lability. The increase in ECD fragment intensity with interface voltages is in part due to improved ion transmission through the skimmer aperture $[25,27]$; in addition, it is probable that "supplemental activation" $[5,35]$ of chargereduced precursors occurs in the interface and increases the yield of ECD products, as evidenced in Figure 5 by the decreased intensity of $(\mathrm{M}+3 \mathrm{H})^{+\cdot \bullet}$ ions at the higher DP value.

\section{Phosphopeptide}

To investigate the suitability of the source for modified peptides, the phosphopeptide UOM9 was analyzed. The source temperature was $200{ }^{\circ} \mathrm{C}$. Figure 6 shows the APECD spectrum of UOM9, acquired with DP alternating between 40 and $80 \mathrm{~V}(50 / 50$ time split), to maximize the yield of ECD fragments over a wide $\mathrm{m} / \mathrm{z}$ range. The spectrum contains an abundance of ECD fragments, including a complete series of $c$-type ions, $c_{3}{ }^{\prime}-c_{10}{ }^{\prime}$, as well as a variety of $z$-type ions, including $z^{*}$ radicals and their oxygen adducts, $z^{*}[36]$, and several even-electron $z^{\prime}$ ions having an extra hydrogen. Of the three varieties of $z$-type ions, the $z^{*}$ ions predominate, forming a near-complete series, $z_{3}{ }^{*}-z_{8}{ }^{*}$, $z_{10}{ }^{*}$, with only $z_{2}{ }^{*}$ missing, presumably because it is collisionally dissociated to form the abundant $z_{2}{ }^{*}$ species (the other missing fragment, $z_{9}{ }^{*}$, is not expected because it requires cleavage on the N-terminal side of proline). Also, due to the low DP value used during half of the scan time, numerous doubly-charged ECD fragments are present, including $c_{6}{ }^{2+}-c_{10}{ }^{\prime 2+}$, as well as a chemical background at low $\mathrm{m} / \mathrm{z}$ (including a prominent peak from dibutylphthalate at $m / z 279$, marked with an asterisk). Most important from Figure 6, however, is that the phosphorylation is retained on all the fragments, providing a first indication that the present AP-ECD source and method can be suitable for analyzing peptides with labile modifications, even when the source temperature is high to activate the precursors and maximize efficiency.

\section{Conclusions}

The AP-ECD source introduced here is capable of providing quality results from low fmol quantities of sample, and it is suitable for modified peptides. Our next research priority is to couple AP-ECD with capillary LC and then apply the method to a wide range of peptides, to determine the oncolumn sensitivity of the method as well as the scope of its potential applications.

\section{Acknowledgments}

The authors gratefully acknowledge funding from the Natural Sciences and Engineering Research Council of Canada (NSERC) and the University of British Columbia (UBC). They also thank Kenny Bach of the UBC Chemistry Department's Mechanical Engineering Services shop for his excellent machining work, Peter Jacobs (Oss, The Netherlands) for his kind donation of the PhotoSpray source, and Larry Campbell of AB SCIEX for helpful discussions.

\section{References}

1. Zubarev, R.A., Kelleher, N.L., McLafferty, F.W.: Electron Capture Dissociation of Multiply Charged Protein Cations. A Nonergodic Process. J. Am. Chem. Soc. 120, 3265-3266 (1998)

2. Zubarev, R.A., Horn, D.M., Fridriksson, E.K., Kelleher, N.L., Kruger, N.A., Lewis, M.A., Carpenter, B.K., McLafferty, F.W.: Electron Capture Dissociation for Structural Characterization of Multiply Charged Protein Cations. Anal. Chem. 72, 563-573 (2000)

3. McLafferty, F.W., Horn, D.M., Breuker, K., Ge, Y., Lewis, M.A., Cerda, B., Zubarev, R.A., Carpenter, B.K.: Electron Capture Dissociation of Gaseous Multiply Charged Ions by Fourier-Transform Ion Cyclotron Resonance. J. Am. Soc. Mass Spectrom. 12, 245-249 (2001)

4. Cooper, H.J., Hakansson, K., Marshall, A.G.: The Role of Electron Capture Dissociation in Biomolecular Analysis. Mass Spectrometry Reviews 24, 201-222 (2005)

5. Syka, J.E.P., Coon, J.J., Schroeder, M.J., Shabanowitz, J., Hunt, D.F.: Peptide and Protein Sequence Analysis by Electron Transfer Dissociation Mass Spectrometry. Proc. Natl. Acad. Sci. U.S.A. 101, 9528-9533 (2004)

6. Mikesh, L.M., Ueberheide, B., Chi, A., Coon, J.J., Syka, J.E.P., Shabanowitz, J., Hunt, D.F.: The Utility of ETD Mass Spectrometry in Proteomic Analysis. Biochim. Biophys. Acta 1764, 1811-1822 (2006)

7. Wiesner, J., Premsler, T., Sickmann, A.: Application of Electron Transfer Dissociation (ETD) for the Analysis of Post-Translational Modifications. Proteomics 8, 4466-4483 (2008)

8. Simons, J.: Mechanisms for $\mathrm{S}-\mathrm{S}$ and $\mathrm{N}-\mathrm{C}_{\mathrm{a}}$ Bond Cleavage in Peptide ECD and ETD Mass Spectrometry. Chem. Phys. Lett. 484, 81-95 (2010)

9. Hoffman, M.D., Sniatynski, M.J., Kast, J.: Current Approaches for Global Post-Translational Modification Discovery and Mass Spectrometric Analysis. Anal. Chim. Acta 627, 50-61 (2008)

10. Voinov, V.G., Deinzer, M.L., Barofsky, D.F.: Radio-Frequency-Free Cell for Electron Capture Dissociation in Tandem Mass Spectrometry. Anal. Chem. 81, 1238-1243 (2009)

11. Voinov, V.G., Deinzer, M.L., Beckman, J.S., Barofsky, D.F.: Electron Capture, Collision-Induced, and Electron Capture-Collision Induced Dissociation in Q-TOF. J. Am. Soc. Mass Spectrom. 22, 607-611 (2011) 
12. Delobel, A., Halgand, F., Laffranchise-Gosse, B., Snijders, H., Laprévote, O.: Characterization of Hydrophobic Peptides by Atmospheric Pressure Photoionization-Mass Spectrometry and Tandem Mass Spectrometry. Anal. Chem. 75, 5961-5968 (2003)

13. Debois, D., Giuliani, A., Laprévote, O.: Fragmentation induced in atmospheric pressure photoionization of peptides. J. Mass Spectrom. 41, 1554-1560 (2006)

14. Robb, D.; Rogalski, J.; Kast, J.; Blades, M. In-Source Atmospheric Pressure-Electron Capture Dissociation (AP-ECD): A New Tool for Structural Characterization of Peptides. Proceedings of the 57th ASMS Conference; Philadelphia, PA, 2009.

15. Rogalski, J. C.; Robb, D.; Blades, M.; Kast, J. In-Source Atmospheric Pressure-Electron Capture Dissociation (AP-ECD): Applicability for the Study of Post-Translational Modifications. Proceedings of the 57th ASMS Conference; Philadelphia, PA, 2009.

16. Robb, D.; Rogalski, J.; Kast, J.; Blades, M. Progress in the Development and Characterization of In-source Atmospheric Pressure-Electron Capture Dissociation (AP-ECD). Proceedings of the 58th ASMS Conference; Salt Lake City, UT, 2010.

17. Berkout, V. M.; Doroshenko, V. M. ECD-like Peptide Fragmentation at Atmospheric Pressure. Proceedings of the 58th ASMS Conference; Salt Lake City, UT, 2010.

18. Robb, D.B., Rogalski, J.C., Kast, J., Blades, M.W.: Atmospheric pressureelectron capture dissociation of peptides using a modified PhotoSpray ion source. Rapid Commun. Mass Spectrom. 24, 3303-3308 (2010)

19. Bagag, A., Giuliani, A., Laprévote, O.: Atmospheric Pressure Photoionization of Peptides. Int. J. Mass Spectrom. 299, 1-4 (2011)

20. Robb, D.B., Covey, T.R., Bruins, A.P.: Atmospheric Pressure Photoionization: An Ionization Method for Liquid Chromatography-Mass Spectrometry. Anal. Chem. 72, 3653-3659 (2000)

21. AB SCIEX PhotoSpray Ion Source-Operator's Manual (Document Number: D1000016276 B). December 2002.

22. Robb, D.B., Blades, M.W.: An Electropneumatic-Heated Nebulizer for Enhancing Spray Ionization in PhotoSpray APPI Sources for LC/MS. Rapid Commun. Mass Spectrom. 23, 3394-3400 (2009)

23. Purves, R.W., Guevremont, R.: Electrospray Ionization High-Field Asymmetric Waveform Ion Mobility Spectrometry-Mass Spectrometry. Anal. Chem. 71, 2346-2357 (1999)

24. AB SCIEX QSTAR XL System-Hardware Guide (P/N: 1003873A). July 2002.

25. Bruins, A.P.: In: Cole, R.B. (ed.) Electrospray Ionization Mass Spectrometry, pp. 107-136. John Wiley and Sons, New York, NY (1997)
26. Loo, J.A., Udseth, H.R., Smith, R.D.: Collisional Effects on the Charge Distribution of Ions from Large Molecules. Formed by Electrosprayionization Mass Spectrometry. Rapid Commun. Mass Spectrom. 2, 207 (1988)

27. Smith, R.D., Loo, J.A., Barinaga, C.J., Edmonds, C.G., Udseth, H.R.: Collisional Activation and Collision-Activated Dissociation of Large Multiply Charged Polypeptides and Proteins Produced by Electrospray Ionization. J. Am. Soc. Mass Spectrom. 1, 53-65 (1990)

28. Kjeldsen, F., Haselmann, K.F., Budnik, B.A., Jensen, F., Zubarev, R. A.: Dissociative Capture of Hot $(3-13 \mathrm{eV})$ Electrons by Polypeptide Polycations: An Efficient Process Accompanied by Secondary Fragmentation. Chem. Phys. Lett. 356, 201-206 (2002)

29. Axelsson, J., Palmblad, M., Hakansson, K., Hakansson, P.: Electron Capture Dissociation of Substance P Using a Commercially Available Fourier Transform Ion Cyclotron Resonance Mass Spectrometer. Rapid Commun. Mass Spectrom. 13, 474-477 (1999)

30. Horn, D.M., Ge, Y., McLafferty, F.W.: Activated Ion Electron Capture Dissociation for Mass Spectral Sequencing of Larger (42 kDa) Proteins. Anal. Chem. 72, 4778-4784 (2001)

31. Mihalca, R., Kleinnijenhuis, A.J., McDonnell, L.A., Heck, A.J.R., Heeren, R.M.A.: Electron Capture Dissociation at Low Temperatures Reveals Selective Dissociations. J. Am. Soc. Mass Spectrom. 15, 18691873 (2004)

32. Lin, C., Cournoyer, J.J., O'Connor, P.B.: Probing the Gas-Phase Folding Kinetics of Peptide Ions by IR Activated DR-ECD. J. Am. Soc. Mass Spectrom. 19, 780-789 (2008)

33. Pitteri, S.J., Chrisman, P.A., McLuckey, S.A.: Electron-Transfer Ion/Ion Reactions of Doubly Protonated Peptides: Effect of Elevated Bath Gas Temperature. Anal. Chem. 77, 5662-5669 (2005)

34. Ledvina, A.R., McAlister, G.C., Gardner, M.W., Smith, S.I., Madsen, J. A., Schwartz, J.C., Stafford Jr., G.C., Syka, J.E.P., Brodbelt, J.S., Coon, J.J.: Infrared Photoactivation Reduces Peptide Folding and HydrogenAtom Migration following ETD Tandem Mass Spectrometry. Angew. Chem. Int. Ed. 48, 8526-8528 (2009)

35. Swaney, D.L., McAlister, G.C., Wirtala, M., Schwartz, J.C., Syka, J.E. P., Coon, J.J.: Supplemental Activation Method for High-Efficiency Electron-Transfer Dissociation of Doubly Protonated Peptide Precursors. Anal. Chem. 79, 477-485 (2007)

36. Xia, Y., Chrisman, P.A., Pitteri, S.J., Erickson, McLuckey, S.A.: Ion/ Molecule Reactions of Cation Radicals Formed from Protonated Polypeptides via Gas-Phase Ion/Ion Electron Transfer. J. Am. Chem. Soc. 128, 11792-11798 (2006) 